\title{
Bridging Reciprocal and Direct Space by Using the Small-Angle Scattering Formalism for Atom Probe Tomography
}

\author{
Frédéric De Geuser ${ }^{1 *}$ \\ 1. Univ. Grenoble Alpes, CNRS, Grenoble INP, SIMaP, F-38000, Grenoble, France. \\ * Corresponding author: frederic.de-geuser@simap.grenoble-inp.fr
}

Small-angle scattering (SAS) has been around as a characterization method for metallurgist for about 70 years [1]. Using mostly X-rays (SAXS) or neutrons (SANS), it is well adapted for the study of phase separation (e.g. precipitation) since it detects inhomogeneities in scattering factor density (i.e. composition). It is capable of providing size and volume fraction of precipitates [2]. Since it is nondestructive, it is well adapted to in situ experiments, providing valuable information on the kinetics of the processes, and essential inputs to modeling [3]. As a diffraction experiment, however, it needs a direct space interpretation model in order to provide structural parameters, and chemistry of the phases to provide volume fractions. It is thus widely used in combination of transmission electron microscopy (TEM) and/or atom probe tomography (APT).

APT is a direct space technique which provides the position of the atoms of each species in a small volume [4]. It gives the local chemistry of each phase, as well as information on the size and shape. It is, however, a destructive and time-consuming technique which does not allow for in situ experiments, and only probes a very small volume. As such, it is a perfect complement to SAS and many studies have used both techniques with great success (e.g. [5]).

In the $80 \mathrm{~s}$, when an atom probe result was essentially a 1D concentration profile, authors have used tools such as autocorrelograms to directly compare their results to SAS [6]. Since the rise of the 3D instruments, however, correlation functions ("RDFs" in the APT jargon) have been arguably underexploited. In the last few years, some efforts have been put into rationalizing the formalism for a better comparison with SAS results [7]. The dual relationship between direct and reciprocal space can be expressed with correlation functions $[8,9]$ :

$$
\begin{gathered}
I(q)=\int_{0}^{\infty} 4 \pi r^{2} \gamma(r) \frac{\sin q r}{q r} d r \\
\gamma(r)=\frac{1}{2 \pi^{2}} \int_{0}^{\infty} I(q) q^{2} \frac{\sin q r}{q r} d q
\end{gathered}
$$

In these equations, $I(q)$, the SAS intensity, and $\gamma(r)$, the correlation function, are Fourier transform pairs, and $r$ and $q$ are the length and spatial frequency variables. For SAS, $\gamma(r)$ is a global function, but APT can separate each element so that it is advantageously written as a sum of partial correlation functions. In addition, SAS is sensitive to each element scattering factor, which should be included in the definition. A detailed expression of the correlation functions can be found in $[7,10]$.

Once the common formalism for both techniques is established, information can travel both ways. Evident input from APT to SAS include:

- Direct space interpretation model (shape, distribution, chemistry of objects)

- Role of each element, redistribution (SAS is mostly "color blind")

- Validation of hypotheses (in particular pseudo-binary assumption, i.e. single type of objects) 
Evident input from SAS to APT include:

- Vastly improved statistics

- In situ experiments

The additional and subtler input from SAS to APT originating from a common formalism is two-fold:

- SAS has essentially no geometric bias and huge probed volumes compared to APT. This means that direct comparison of well-defined sizes or distances obtained in SAS and APT could be used as an aid for calibration of APT reconstructions, better understanding of local magnification issues or estimation of effective spatial resolution

- The SAS traditional data processing strategy (i.e. parametric model fitting the SAS intensity) can be equally applied to the direct space correlation functions ("RDFs"), providing a reliable average estimate of size and volume fraction of objects in an APT volume. This approach is essentially parameter-free [10] which is in contrast with the traditional cluster analysis methods used in APT.

In summary, the definition of a common formalism between APT and SAS allows for more efficient combined studies. In addition, it provides the APT community with the SAS set of data interpretation techniques, which can be equally applied in direct space, and provide averages metrics on the microstructure included in the APT volume.

References:

[1] A. Guinier, G. Fournet, Small-angle scattering of X-rays, John Wiley \& Sons, New York, 1955.

[2] G. Kostorz, Z. Für Krist. - Cryst. Mater. 218 (2003).

[3] A. Deschamps, C. Sigli, T. Mourey, F. De Geuser, W. Lefebvre, B. Davo, Acta Mater. 60 (2012) 1917-1928.

[4] B. Gault, M.P. Moody, J.M. Cairney, S.P. Ringer, Atom Probe Microscopy, (Springer-Verlag, New York), 2012.

[5] M. Miller, B. Wirth, G. Odette, Mater. Sci. Eng. -Struct. Mater. Prop. Microstruct. Process. 353 (2003) 133-139.

[6] S. Spooner, M.K. Miller, J. Phys. Colloq. 49 (1988) C6-405-C6-410.

[7] L. Couturier, F. De Geuser, A. Deschamps, Mater. Charact. 121 (2016) 61-67.

[8] P. Debye, A.M. Bueche, J. Appl. Phys. 20 (1949) 518-525.

[9] G. Porod, General Theory, in: O. Glatter, O. Kratky (Eds.), Small-Angle X-Ray Scatt., (Academic Press, London), 1982, p. 17-51.

[10] H. Zhao, B. Gault, D. Ponge, D. Raabe, F. De Geuser, Scr. Mater. 154 (2018) 106-110. 